\title{
Visualizing recombination intermediates with single-stranded DNA curtains
}

Zhi $\mathrm{Qi}^{1 \dagger}$ and Eric C. Greene ${ }^{1 *}$

${ }^{1}$ Department of Biochemistry \& Molecular Biophysics, Columbia University, New York, NY

*email: ecg2108@cumc.columbia.edu

${ }^{\dagger}$ Present Address: Center of Qualitative Biology \& Center of Life Sciences, Academy for Advanced Interdisciplinary Studies, Peking University, Beijing, China, 100871

\section{Key words:}

Homologous recombination; RecA, Rad51, Presynaptic complexes; Homology search; Singlemolecule approaches; Total internal reflection fluorescence microscopy.

\begin{abstract}
Homologous recombination (HR) is a critical cellular process for repairing double-stranded DNA breaks (DSBs) - a toxic type of DNA lesion that can result in chromosomal rearrangements and cancer. During the early stages of HR, members from the Rad51/RecA family of recombinases assemble into long filaments on the single-stranded DNA overhangs that are present at processed DSBs. These nucleoprotein filaments are referred to as presynaptic complexes, and these presynaptic complexes must align and pair homologous DNA sequences during HR. Traditional ensemble methods cannot easily access the transient and often heterogeneous intermediates that are typical of DNA recombination reactions, and as a consequence, there remain many open questions with respect to the molecular details of this pathway. Novel single-molecule approaches that are capable of directly visualizing reaction intermediates in solution and in real time offer the potential for new insights into the mechanism of homologous DNA recombination. Here we highlight recently developed single stranded DNA curtain methods for studying the properties of individual Rad51 presynaptic complexes and other related recombination intermediates at the single-molecule level.
\end{abstract}




\section{Introduction}

Genomes can be easily damaged by exogenous agents, like ionizing radiation, and endogenous processes, such as DNA replication [1]. Broken DNA can affect genome stability, potentially leading to the onset of cancers and other genetic diseases [2]. Double-stranded DNA breaks (DSBs) (Fig. 1) are highly cytotoxic lesions, and if left unrepaired, they can lead to cell death or gross chromosomal rearrangements that are a hallmark of most forms of cancer [3]. To preserve genome integrity, cells possess several DNA repair pathways. Of these pathways, homologous recombination (HR) offers an error-free solution - meaning it causes little or no alteration in the original DNA sequence - to repair DSBs. A key feature of HR is that it uses a homologous DNA sequence as a template to guide repair of broken chromosomes [4] (Fig. 1).

$\mathrm{HR}$ is promoted by the members of the RecA/Rad51 family of recombinases - RecA in bacteria, RadA in archaea, Rad51/Dmc1 in eukaryota, and UvsX in bacteriophages [5-7]. These proteins bind to the single-stranded DNA overhangs present at the processed ends of DSBs, yielding the presynaptic complex, which is a key intermediate in homologous recombination [5, 7]. The presynaptic complex must first search through vast amounts of genomic DNA (Fig. 1) to find the specific complementary DNA sequence required to repair a particular DSBs. This process is referred to as the "homology search" [8,9]. Once the correct homologous DNA sequence is located, the presynaptic complex can invade the duplex DNA sequence (Fig. 1), forming an intermediate called displacement-loop (D-loop)[5, 7]. This step of the reaction is referred to as strand invasion, and the resulting D-loop intermediate then sets the stage for proteins involved in the downstream steps in the reaction to complete the repair process [10-12].

RecA is considered the prototypical member of the Rad51/RecA family DNA recombinases and many of the general properties of RecA are conserved among other members of the $\operatorname{Rad} 51 / \operatorname{Rec} A$ family [5]. RecA is an ATP-dependent DNA-binding protein that assembles into long filaments on ssDNA [5, 13, 14]. Although RecA can hydrolyze ATP, it can also bind ssDNA in the presence of the non-hydrolyzable ATP analogue ATP $\gamma$, resulting in a stable presynaptic that can perform strand invasion without extensive ATP hydrolysis. Remarkably, the ssDNA bound by RecA is extended by $\sim 50 \%$ relative to an equivalent length of B-form DNA. However, structural studies have revealed that the ssDNA is not uniformly extended, instead the ssDNA is organized into base triplets, which have near B-form geometry, while the phophodiester backbone is stretched to $\sim 8 \AA$ between each base triplet giving rise to an overall extension of the DNA molecule [15]. We refer this unique DNA architecture as RS-DNA (recombinase-stretched DNA) to reflect its unique structural [15] and functional characteristics $[16,17]$, and to distinguish it from other forms of stretched DNA, such as S-DNA [18-20]. This unique structural organization of RS-DNA has important implications for understanding how RecA/Rad51 filaments assemble and how they promote DNA strand invasion.

Cellular methods, like cell biology and genetics, and molecular methods, such as biochemistry, molecular biology, and structural biology, have made tremendous progress in understanding presynaptic complex composition and assembly. However, many molecular details of presynaptic complex behavior are still lacking. Single-molecule approaches are now beginning to offer new insights into recombination that cannot be easily accessed by other approaches [21-24]. In contrast to traditional techniques, single-molecule approaches can be used to directly visualize transient biochemical intermediates and heterogeneous reactions in unprecedented detail. Here we discuss recently established approaches for studying the 
properties of presynaptic complexes using single-stranded DNA curtains and total internal reflection fluorescence microscopy.

\section{Single-stranded DNA curtains}

DNA curtains provide a flexible experimental platform that can be used for studying various protein-nucleic acid interactions, by enabling the direct visualization of hundreds to thousands of individual binding reactions in real-time [25]. DNA curtains can be made with either doublestranded DNA [26, 27] or single-stranded DNA [28], and the ssDNA curtains now offer the unique possibility to visualize reactions on long ssDNA substrates (Fig. 2). Below we provide brief examples of how ssDNA curtains can be used to study the properties of Rad51 presynaptic complexes, and in Section 3 we will describe step-by-step procedures for preparing ssDNA curtains and assembling Rad51 presynaptic complexes.

\subsection{DNA curtains and presynaptic complex assembly}

Biotinylated ssDNA molecules are first prepared by rolling circle DNA replication (Fig. 2A), and these ssDNA substrates are then injected into a preassembled microfluidic flowcell. The flowcell surface is coated with a fluid lipid bilayer, and a subset of the lipids are covalently labeled with biotin, allowing the ssDNA to be anchored to the surface via a biotin-streptavidin linkage (Fig. 2B). Buffer containing an eGFP-tagged version of replication protein A (RPA) is then injected into the sample chamber. RPA is a ubiquitous eukaryotic ssDNA binding protein that is involved in all aspects of DNA metabolism involving an ssDNA intermediate [29, 30]. The fluorescent RPA-eGFP binds to the ssDNA and promotes the removal of secondary structures, allowing the ssDNA to be visualized by fluorescence microscopy. Buffer flow is then used to push the RPA-ssDNA complexes into chromium barriers that are located on the surface of the slide (Fig. 2B). The chromium barriers disrupt the continuity of the lipid bilayer and block any further movement of the anchored ssDNA along the surface, as a result, all of the ssDNARPA complexes align at the leading edges of these barriers (Fig. 2B). When viewed by widefield total internal reflection fluorescence microscopy (TIRFM), these RPA-ssDNA complexes give the appearance of a "curtain" of aligned complexes (Fig. 3A). For a single-tethered format, the downstream 3' ends of the RPA-ssDNA complexes are left free in solution [31, 32], whereas in a double-tethered format the 3' ends of the RPA-ssDNA are nonspecifically adsorbed to exposed anchor points located downstream from the chromium barriers (Fig. 2B \& Fig. 3A) [16, 17]. The primary advantage of the double-tethered format is that it allows for continuous observation of the long ssDNA substrates even when buffer flow is terminated [33].

The RPA-ssDNA complexes serve as the platform for assembly of the Rad51 presynaptic complex (Fig. 1, Fig. 2B \& Fig. 3B). To initiate presynaptic complex assembly, S. cerevisiae Rad51 is injected into the flowcell along with buffer containing the required nucleotide cofactor, but lacking any additional free RPA-eGFP. Rad51 binds to the ssDNA, resulting in the displacement of the fluorescent RPA-eGFP molecules, as a consequence, successful presynaptic complex assembly coincides with the loss of the eGFP fluorescence signal (Fig. 3). An important advantage of this approach is that it does not require fluorescently tagged Rad51 protein. The resulting data are readily displayed in the form of a kymograph (Fig. 3B-F), which represents a single pixel slice through just one ssDNA molecule from within the curtain and can be 
interpreted based on the fluorescence signal. For instance, in Fig. 3B, at the outset of the experiment the ssDNA is bound by RPA-eGFP, yielding a strong green fluorescent signal. Rad51 and ATP are then injected into the flowcell and this injection coincides with the rapid loss of the green RPA-eGFP signal as unlabeled (dark) Rad51 displaces the fluorescent RPA-eGFP from the ssDNA. At this stage of the reaction the free Rad51 can be removed from solution so long as ATP (or an appropriate ATP analog) remains present in the buffer (Fig. 3B \& Fig. 3F). In contrast, if the ATP present during the initial assembly of the Rad51 presynaptic complex is removed and replaced with buffer lacking nucleotide cofactor (Fig. 3C) or containing ADP (Fig. 3E) then the Rad51 filaments rapidly dissociate as revealed by the reappearance of the fluorescent RPA-eGFP signal on the ssDNA.

\subsection{Recombination accessory proteins}

The simplified schematic of homologous recombination presented in Fig. 1 only highlights the participation of two proteins, RPA and Rad51, during presynaptic complex assembly. However, many other proteins are necessary for recombination in living cells, including: recombination mediators, such as Rad52 and Brca2, which promote the assembly of the Rad51 filament [10, 34]; helicases, such as Srs2, Sgs1, Rad54, and Mph1, which perform varied functions during recombination, including roles in end resection, strand invasion, and dissolution of inappropriate recombination intermediates [35, 36]; finally, there are Rad51 paralogs, such as Rad55/57 and the SHU complex, whose functions remain poorly understood, but recent data indicates that they seem to participate in stabilizing the presynaptic complex [37-39]. Experiments using ssDNA curtains offer the potential for studying these types of recombination accessory factors to gain deeper insights into how they interact with and affect the properties of the Rad51 presynaptic complex. For example, using ssDNA curtains we have begin to dissect the interactions between ssDNA, RPA, Rad52, and Rad51 during presynaptic complex assembly (Fig. 4) [31, 32, 40]. Using RPA-mCherry and a SNAP-Rad52 fusion construct labeled with an Alexa 488 fluorophore we were able to directly visualize the binding interactions between Rad52 and the RPA-ssDNA complexes (Fig. 4A). Wide-field TIRFM imaging revealed these interactions as green Rad52 proteins co-localized with the red-emitting RPA-mCherry complexes (Fig. 4B-C). Kymographs of experiments conducted in real-time were used to reveal the binding kinetics of Rad52 to the RPA-ssDNA complexes (Fig. 4D), and similar approaches were used to measure binding distributions, assembly mechanisms, and lifetimes of the assembled complexes [32]. These examples highlight the tremendous potential for the use of ssDNA curtains in conjunction with fluorescently-tagged proteins to begin establishing a more in-depth understanding of how recombination accessory proteins interact with the Rad51 presynaptic complexes and other recombination intermediates.

\subsection{Duplex DNA capture and the homology search mechanism}

A key characteristic of the presynaptic complex is its ability to align and pair homologous DNA sequences. To fulfill this function the Rad51 protein filament must be capable of interacting with two different DNA molecules: the ssDNA present at processed DSBs, which serves are the substrate for presynaptic complex assembly, and a homologous dsDNA sequence, which is required to serve as a template for repair. The interactions between these two different DNA 
molecules are governed by two conceptually distinct binding surfaces on the protein filament, referred to as the primary and secondary binding sites $[5,7,15]$. The primary binding site interacts with the presynaptic ssDNA and the secondary binding site is thought to allow interactions with the duplex DNA as the presynaptic complex searches for homology and attempts to promote strand invasion. Thus, the ability of the Rad51-ssDNA presynaptic complex to sample and bind to duplex DNA sequences is essential to homologous recombination, but these interactions are difficult to study because of the transient and heterogeneous nature of the intermediates involved in both the homology search and strand invasion.

In recently published work, we demonstrated that ssDNA curtains can be used to study both the homology search and the process of strand invasion [16, 17]. These experiments rely upon the use of short duplex DNA fragments (70-base pairs, bp) containing various lengths of sequence microhomology (see Section 5.4) labeled at one end with a single fluorescent dye. These DNA fragments are injected into a flowcell sample chamber containing preassembled Rad51 presynaptic complexes allowing the detection of two general classes of intermediates: (i) transient binding intermediates that can be detected when the reactions are viewed in real time; and (ii) longer-lived intermediates that remain bound even after the free dsDNA fragments are flushed from the sample chamber (Fig. 5A-C). The transiently bound intermediates reflect processes that take place during the homology search (Fig. 5B), and the more stably bound DNA fragments represent strand invasion intermediates (Fig. 5C). The lifetimes of both types of intermediates can be quantitatively analyzed by determining the dwell times of the observed binding events and graphing the resulting data as survival probability plots (Fig. 5D, see Section 5.2). These types of experiments using dsDNA fragments have revealed several new features of the homology search and strand invasion reactions. First, analysis of the transient binding events revealed that the homology search takes place through a process that exhibits a characteristic power law behavior, suggesting the existence of binding intermediates encompassing a broad range of stabilities [17]. Second, these experiments have revealed the existence of a lengthspecific selection mechanism that allows the Rad51 presynaptic complex to kinetically ignore nonhomologous tracts of duplex DNA that are shorter than 8-nucleotides in length, ensuring that the search process is focused only on longer ( $\geq 8-n t)$ tracts of microhomology, which have a greater probability of being the correct sequence [17]. Third, analysis of the stable binding intermediates reveals that strand exchange intermediates are stabilized in 3-nucleotide increments, which reflects the unique base triplet structural organization of the RS-DNA bound within the presynaptic complex [16]. We anticipate that future studies of the interactions between the presynaptic complex and dsDNA molecules will continue to reveal new insights into these reactions.

\section{Preparation of ssDNA curtains}

Here we provide a step-by-step guide describing all of the general procedures necessary for preparing ssDNA curtains, assembling Rad51 presynaptic complexes, as well as basic aspects of experimental design for studying the properties of the presynaptic complexes.

\subsection{Rolling circle DNA replication}

Rolling circle replication (RCR) (Fig. 2B) is used to produce the long ssDNA substrates necessary for making ssDNA curtains $[17,28]$. A biotinylated primer $\left(5^{\prime}\right.$ - BIOTEG - TTT TTT 
TTT TTT TTT TTT TTT TTT TTT TTT - GTA AAA CGA CGG CCA GT) is annealed to a M13 ssDNA circle template (M13mp18, 7,249 nt, N4040S NEB) to form the RCR template. Replication is initiated by the addition of $\Phi 29$ DNA polymerase in the presence of deoxynucleotide (dNTP, N0447L NEB), resulting in the production of a long ssDNA molecule comprised of repeating units of M13mp18.

Preparation of the RCR template:

Step 1. Prepare a $100 \mu \mathrm{l}$ annealing reaction containing the following: (i) $10 \mu \mathrm{l} 10 \times$ Annealing buffer (400 mM Tris- $\mathrm{HCl}[\mathrm{pH} 8.0], 500 \mathrm{mM} \mathrm{NaCl}$, and $100 \mathrm{mM} \mathrm{MgCl}$ ); (ii) $40 \mu \mathrm{l}$ M13mp18 (112 nM, MW $2236557.3 \mathrm{~g} / \mathrm{mol}$; corresponding to a final concentration of $44.8 \mathrm{nM}$ ); (iii) $1.8 \mu \mathrm{l}$ primer $(2 \mu \mathrm{M}$, MW $14835.8 \mathrm{~g} / \mathrm{mol}$; corresponding to a final concentration of $36 \mathrm{nM}$ ), yielding a template to primer ratio of $1.2: 1$; (iv) add $48.2 \mu \mathrm{l}$ distilled water, bring the total volume of the reaction to $100 \mu$ l.

Step 2. Heat the reaction mixture to $90^{\circ} \mathrm{C}$ for 5 minutes in a one liter beaker containing $800 \mathrm{ml}$ water, and then place the beaker on a lab bench and allow it to slowly cool at room temperature for a period of four hours.

Step 3. Add $260 \mu \mathrm{l}$ EB Buffer (10 mM Tris- $\mathrm{HCl}[\mathrm{pH} 8.5])$ to dilute the annealing primertemplate product to a final concentration of $10 \mathrm{nM}$.

Step 4. The final sample can then be stored at $4^{\circ} \mathrm{C}$ prior to use.

Protocol for rolling circle DNA replication:

Step 1. Prepare a $50 \mu 1$ RCR containing: (i) $10 \mu 15 \times$ RCR buffer ( $250 \mathrm{mM}$ Tris-HCl [pH 7.5], 20 $\mathrm{mM}$ DTT, $50 \mathrm{mM}$ ammonium sulfate, and $50 \mathrm{mM} \mathrm{MgCl}$ ); (ii) $2 \mu$ primer-M13mp18 complex (10 nM; for a final concentration of $0.4 \mathrm{nM}$ ); (iii) $2 \mu \mathrm{l} \Phi 29$ DNA polymerase (10 $\mu \mathrm{M}$ stock; corresponding to a final concentration of $0.4 \mu \mathrm{M})$; (iv) $2 \mu \mathrm{lNTP}(10 \mathrm{mM}$; corresponding to a final concentration of $0.4 \mathrm{mM}$ ); (v) add $34 \mu \mathrm{l}$ distilled water to bring the reaction volume to $50 \mu \mathrm{l}$.

Step 2. Incubate the RCR mixture at $30^{\circ} \mathrm{C}$ for $\sim 25$ minutes, and then dilute the mixture by the addition of $900 \mu \mathrm{l}$ BSA buffer ( $40 \mathrm{mM}$ Tris- $\mathrm{HCl}$ [pH 7.5], $2 \mathrm{mM} \mathrm{MgCl} 2,1 \mathrm{mM}$ DTT, and $0.2 \mathrm{mg} / \mathrm{ml} \mathrm{BSA}$ ). This diluted solution is ready to be injected into a flowcell for ssDNA curtains experiments.

Tips \& Troubleshooting: (i) Clean distilled water is a key to generating good ssDNA substrates from RCR; (ii) The annealed RCR primer-template can be stored at $4^{\circ} \mathrm{C}$, but should be freshly prepared every two months.

\subsection{Lipid bilayer preparation}

Lipid bilayer components come from Avanti Polar Lipids: (i) DOPC (1,2-dioleoyl-sn-glycero-3phosphocholine), 1 g powder; (ii) PEGylated lipids (18:1 PEG2000 DOPE, 1,2-dioleoyl-snglycero-3-phosphoethanolamine-N-[methoxy (polyethylene glycol) - 2000] (ammonium salt)), $100 \mathrm{mg}$ powder; (iii) biotinylated lipids (18:1 Biotinyl Cap DOPE, 1,2-dioleoyl-sn-glycero-3phosphoethanolamine-N-(cap biotinyl) (sodium salt)), $25 \mathrm{mg}$ powder. 
Preparation of the lipid stock:

Step 1. Dissolve $1 \mathrm{~g}$ DOPC (100 mg/ml), $100 \mathrm{mg}$ PEG-2000 DOPE (10 mg/ml), and $5 \mathrm{mg}$ biotinylated DOPE $(0.5 \mathrm{mg} / \mathrm{ml})$ into $10 \mathrm{ml}$ of chloroform.

Step 2. The lipid stock is divided into $1 \mathrm{ml}$ aliquots and stored at $-20^{\circ} \mathrm{C}$. The storage vials are sealed with parafilm.

Preparation of the liposomes:

Step 1. Clean a $2 \mathrm{ml}$ glass vial (National Scientific C4015-1) and cap (National Scientific CB7815-B) with distilled water and 70\% ethanol, dry with nitrogen gas, and incubate in a drying oven at $150^{\circ} \mathrm{C}$ for 20 minutes.

Step 2. During the incubation, a lipid stock aliquot is removed from storage at $-20^{\circ} \mathrm{C}$ and placed inside a hood at room temperature for $\sim 10$ minutes to prevent water condensation upon opening.

Step 3. Rinse a 250- $\mu 1$ Hamilton glass syringe several times with chloroform, and then transfer $200 \mu \mathrm{l}$ of lipid stock to the clean glass vial using the Hamilton glass syringe.

Step 4. The chloroform is then evaporated with a very weak stream of nitrogen gas with continuous slow rotation of the glass vial by hand. A thin white layer of dried lipid should form on the inside wall of the glass vial.

Step 5. The glass vial is then incubated under vacuum overnight ( $\geq 16$ hours) to ensure complete removal of all chloroform.

Step 6. $2 \mathrm{ml}$ lipid buffer (10 mM Tris-HCl [pH 8.0] and $100 \mathrm{mM} \mathrm{NaCl})$ is then added to dissolve the dried lipids, and the glass vial is then placed at room temperature for at least 2 hours to ensure complete suspension of the lipids.

Step 7. Vortex the sample for 2-3 minutes to form large multilamellar vesicles, At this stage the solution should appear white and cloudy.

Step 8. This solution is transferred to a 5-ml polypropylene culture tube (Falcon 35-2058, Becton Dickinson labware) for sonication (VirSonic micro-tip sonicator) to form small unilamellar vesicles. Sonication is performed at an amplitude setting of 60, with a total process time of 3 minutes using 20 second pulses separated by 20 second intervals. After sonication, the liposome solution becomes translucent.

Step 9. The liposome solution is then filtered through a $0.22-\mu \mathrm{m}$ nylon syringe filter, and then is aliquoted and stored at $4^{\circ} \mathrm{C}$. Do not freeze! The liposome solution can be used for 2 or 3 months when stored at $4^{\circ} \mathrm{C}$.

Tips \& Troubleshooting: (i) During the sonication, the sonication probe should keep below the sample surface. If not, the probe will produce a large amount of bubbles, resulting in damage to the vesicles. (ii) The liposome solution should be kept on ice during sonication to avoid heating the sample. (iii) It is good practice to prepare a fresh liposome solution every two months.

\subsection{Nanofabrication}

The nanofabrication protocol of the silica slides is described in detail in Greene et al. [25]. Here we summarize the most important steps. 
Step 1. Two small holes are drilled on a quartz microscope slide (G. Finkenbeiner INC., 1" x 3" x $1 \mathrm{~mm}$ Thick) using a Servo drill press and diamond-coated drill bit (1.4 mm dia.). A number/designation can be etched on one corner of the slide using a diamond tipped bit to help keep track of the slide and provide the information about the design of the nanofabrication patterns.

Step 2. The quartz microscope slides are cleaned as follows: (i) Sonicate the slides in acetone for 20 minutes; (ii) Sonicate in $1 \mathrm{M} \mathrm{NaOH}$ for 20 minutes; (iii) Rinse the slides in distilled water; (iv) Prepare $200 \mathrm{ml}$ piranha solution $(150 \mathrm{ml}$ sulfuric acid and $50 \mathrm{ml}$ hydrogen peroxide) and then rinse the slides in the piranha solution for 30 minutes; (v) Rinse the slides in distilled water, acetone, and isopropanol, and then dry with nitrogen gas.

Tips \& Troubleshooting: ( $i)$ The piranha solution is very dangerous, and standard protective equipment is required. (ii) Keep the hydrogen peroxide cold by storing at $4^{\circ} \mathrm{C}$. (iii) For the piranha solution preparation, the $50 \mathrm{ml}$ hydrogen peroxide should be poured very slowly into the $150 \mathrm{ml}$ sulfuric acid solution.

Step 3. The quartz microscope slides are coated with the PMMA and AquaSAVE by spin coating. (i) Prepare a $25 \mathrm{kDa}$ polymethylmethacrylate (PMMA) (in anisole (MicroChem, Newton, MA)) solution, a $495 \mathrm{kDa}$ PMMA (in anisole) solution, and a AquaSAVE conducting polymer solution (Mitsubishi Rayon). (ii) Use the following spin coating parameters: 4,000 rpm, $300 \mathrm{rpm} / \mathrm{s} \mathrm{ramp,} \mathrm{for} \mathrm{a} \mathrm{total} \mathrm{time} \mathrm{of} 45$ seconds. (iii) Fix one quartz microscope slide to the stage of the spin coating machine and use a transfer pipet to add 3 or 4 drops of the $25 \mathrm{kDa}$ PMMA solution and start the first spin run; using a new transfer pipet add 3 or 4 drops of the $495 \mathrm{kDa}$ PMMA and then start the second run; use a third transfer pipet to add 2 drops of the Aquasave conducting polymer and start the final run. This procedure yields three layers on the top of the quartz surface: $25 \mathrm{kDa}$ PMMA; $495 \mathrm{kDa}$ PMMA; and AquaSAVE [25].

Step 4. Nanofabrication patterns are designed by using NPGS (Nano Pattern Generator System) software and an FEI E-Beam Writer (E-Beam lithography), which etches through all three layers until reaching the quartz surface [25].

Step 5. The etched patterns need to be cleaned of residual PMMA and AquaSave, as follows: (i) Place the slide into a $50 \mathrm{ml}$ tube with fresh distilled water for several minutes, and then dried with nitrogen gas; (ii) place the slide into a new $50 \mathrm{ml}$ tube with a fresh resist developer solution (MIBK (Methyl Isobutyl Ketone):Isopropanol [1:3]), and then place the $50 \mathrm{ml}$ tube into a water bath sonicator at $4^{\circ} \mathrm{C}$ for 1 minute; (iii) After sonication, quickly remove the slide from the resist developer solution, then wash the slide with isopropanol and dry using a stream of nitrogen gas.

Step 6. Chromium patterns are then deposited on the surface using a Semicore Thermal Evaporation, as previously described [25], to yield a final barrier height of $\sim 25$ nanometers $(\mathrm{nm})$. Note that diffusion barriers of different heights ( 10 to $\sim 300-\mathrm{nm})$ work similarly well.

Step 7. Finally, the $25 \mathrm{kDa}$ and $495 \mathrm{kDa}$ PMMA layers remaining around the chromium patterns are removed, as follows: (i) The slides are first rinsed with acetone; (ii) The slides are then sonicated in acetone for 5 minutes; (iii) The slides are rinsed in distilled water, followed by ethanol, and they are then dried with a stream of nitrogen gas. 
Step 8. The chromium patterns on new slide should be checked with an optical microscope prior to use.

Step 9. Patterned slides can be kept in $50 \mathrm{ml}$ polypropylene tubes and stored under vacuum.

\subsection{Flowcell preparation}

Each flowcell consists of a nanofabricated, fused silica slide (Section 4.3), a thin coverslip, and a piece of double-sided tape that forms the boundaries of the sample chamber (Fig. 3A). Nanoports are glued to two drilled holes on the top silica slide (Quartz microscope slides from G. Finkenbeiner Inc.), one for input and output respectively.

\subsection{Sample delivery}

Our sample delivery system includes syringe pumps ( $\mathrm{kd}$ Scientific) and two injection valves (SCIVEX). DuPont PFA Tubing (IDEX, 1512L, DuPont ${ }^{\circledR}$ PFA Tubing Natural 1/16" OD x .020" ID x 50ft) and the IDEX connecting ports are used to connect all components. A lowpressure 4-port switching valve (V-101D, IDEX) can be used to switch between two different buffer sources when used together with two syringe pumps. For injection, samples are first loaded into a small loop (either $50 \mu 1$ or $500 \mu \mathrm{l}$ ) located on the injection valve. After the buffer flow is initiated, the sample is delivered to the flowcell by pressing "injection" button on the injection valve. Following a short delay, the sample will reach the flowcell and then the buffer flow can be turned off allow the injected reagents (e.g. Rad51 and ATP) to incubate with the ssDNA. Knowing the length of this delay time is a crucial aspect of any experiment in which the buffer flow will be terminated after the injection of a sample. The delay time depends on: (i) the length of tubing between the injection loop and the flowcell; (ii) the volume of the loop; (iii) and the flow rate of the syringe pump.

The protocol for determining the sample delay time is as follows:

Step 1. First, load $1 \mathrm{nM}$ eGFP (any eGFP tagged protein or other fluorophore should suffice) into a 50 or $500 \mu$ sample loop.

Step 2. The syringe pump is turned on at the desired flow rate (we typically use 0.2 to 1.0 $\mathrm{ml} / \mathrm{min}$ ), and initiate data acquisition.

Step 3. After allowing the buffer to flow for $\sim 1$ minute, switch the injection valve to allow delivery of the fluorescent sample to the flowcell while continuously collecting data.

The fluorescence intensity change as the fluorescent sample passes through the flowcell provides a measure the sample waiting time. When measuring the delay time, the "zero" time point reflects the time at which the injection valve is switched to "Injection mode", and the resulting peaks in fluorescence signal reflect passage of the fluorescent sample through the flowcell. The width, height, and position of the resulting signal peak are all factors that should be taken into consideration during experimental design to ensure that the maximum amount of sample is present within the flowcell for the desired observation window.

Tips \& Troubleshooting: The two problems that can be encountered with the injection systems are leaks, which can lead to bubbles, and the presence of contaminants from previous users. To 
avoid these problems be sure to wash the microfluidic system with distilled water before each experiment, pushing out all bubbles and double-check for any loose fittings that may leak. Clean the tubing carefully after the experiment is complete. To clean the tubing, flush the system sequentially with water $(10 \mathrm{ml}), 7 \mathrm{M} \mathrm{Urea}(2 \mathrm{ml})$, water $(10 \mathrm{ml}), 0.1 \mathrm{M} \mathrm{HCl}(2 \mathrm{ml})$, water $(10$ $\mathrm{ml}), 0.1 \mathrm{M} \mathrm{NaOH}(2 \mathrm{ml})$, water $(10 \mathrm{ml})$, and finally wash with $20 \%$ ethanol. The system can be stored in $20 \%$ ethanol and should be flushed with water prior to use.

\subsection{Preparation of the ssDNA curtains}

Step 1. Wash a clean flowcell with $3 \mathrm{ml}$ lipid buffer $(10 \mathrm{mM}$ Tris-HCl $[\mathrm{pH} 8.0]$ and $100 \mathrm{mM}$ $\mathrm{NaCl})$.

Step 2. Inject $1 \mathrm{ml}$ liposomes (40 $\mu$ liposome storage solution (Section 4.2) plus $960 \mu \mathrm{l}$ lipid buffer $(10 \mathrm{mM}$ Tris- $\mathrm{HCl}[\mathrm{pH} 8.0]$ and $100 \mathrm{mM} \mathrm{NaCl}))$ into the flowcell. The $1 \mathrm{ml}$ liposome solution is injected by hand at the bench using a syringe in four sequential steps ( $250 \mu \mathrm{l}$ per step) with an incubation time of $\sim 5$ minutes between each injection.

Step 3. The flowcell is then washed with another $3 \mathrm{ml}$ lipid buffer, and then incubated at room temperature for 30 minutes. This procedure produces a lipid bilayer on the flowcell surface.

Step 4. The flowcell is then washed with $3 \mathrm{ml} \mathrm{BSA}$ buffer $(40 \mathrm{mM}$ Tris- $\mathrm{HCl}$ [pH 7.5], $2 \mathrm{mM}$ $\mathrm{MgCl}_{2}, 1 \mathrm{mM}$ DTT, and $0.2 \mathrm{mg} / \mathrm{ml} \mathrm{BSA}$ ).

Step 5. A $50 \mu 1$ sample of biotinylated ssDNA is freshly prepared using rolling circle replication as described in Section 3, and then this sample is then transferred to a fresh tube containing $750 \mu \mathrm{l}$ of BSA buffer.

Step 6. The lipid bilayer, which contains a small fraction of biotinylated lipids, is then rinsed with $800-\mu l$ streptavidin buffer $(10 \mu \mathrm{l}$ streptavidin stock $(1 \mathrm{mg} / \mathrm{ml})$ plus $790 \mu \mathrm{l}$ BSA buffer). The streptavidin buffer is injected in four steps (200 $\mu 1$ per step), and allowed to incubate for $\sim 5$ minutes between each injection. Note that the streptavidin stock (1 $\mathrm{mg} / \mathrm{ml}$ ) is prepared by dissolving $5 \mathrm{mg}$ streptavidin (Invitrogen A00045) into $5 \mathrm{ml}$ distilled water. This streptavidin stock solution can then be divided into small aliquots and stored at $-20^{\circ} \mathrm{C}$.

Step 7. The flowcell is washed with an additional $3 \mathrm{ml}$ BSA buffer to remove any free streptavidin.

Step 8. The entire $800 \mu \mathrm{l}$ RCR sample from Step 5 is then injected into the flowcell. The RCR sample is injected by hand in four steps (200 $\mu \mathrm{l}$ per step), and the incubation time between injections is $\sim 5$ minutes. After this step the flowcell is installed on the microscope stage and coupled to the sample delivery system (Section 4.2).

Step 9. BSA buffer with $0.1 \mathrm{nM}$ RPA-eGFP is injected into flowcell at a flow rate of $1.0 \mathrm{ml} / \mathrm{min}$ for 15 minutes. The RPA-eGFP binds to the ssDNA and removes secondary structure, and the buffer flow pushes the tethered ssDNA to the leading edges of the chromium barriers. When used in a double-tethered format, chromium pedestals positioned downstream of the barriers provide anchor points for the nonspecific adsorption of the RPA-ssDNA ends (Fig. 2B). An injection pulse of 7 M Urea $(500 \mu \mathrm{l})$ can also be used to remove protein aggregates, residual $\Phi 29$ DNA polymerase, and any remaining secondary structures.

\subsection{Assembly of the presynaptic complex}


Step 1. The RPA-ssDNA complexes are then washed with HR buffer (30 mM Tris-Acetate [pH 7.5], $20 \mathrm{mM} \mathrm{Mg}$-Acetate, $50 \mathrm{mM} \mathrm{KCl}, 1 \mathrm{mM}$ DTT, $0.2 \mathrm{mg} / \mathrm{ml} \mathrm{BSA}$, and $2.5 \mathrm{mM}$ ATP) [41], at a flowrate of $1 \mathrm{ml} / \mathrm{min}$ flow rate for 2 minutes to remove free RPA-eGFP.

Step 2. S. cerevisiae $\operatorname{Rad} 51(1 \mu \mathrm{M})$ is then injected into the flowcell at $30^{\circ} \mathrm{C}$ using a $50 \mu$ sample loop, and flow is turned off once the protein has entered the sample chamber. This incubation step allows Rad51 to exchange with the bound RPA-eGFP resuting in formation of the presynaptic complexes. Similar procedures, with only minor changes to buffer composition and reaction temperature, can be used to prepare presynaptic complexes comprised of human Rad51, human Dmc1, S. cerevisiae Dmc1, and E. coli $\operatorname{Rec} A[16,17]$.

Tips \& Troubleshooting: It is extremely important to avoid injecting bubbles through the flowcell. It is easy to accidentally inject bubbles into the flowcell, and passage of even small bubbles through the flowcell will destroy the quality of the DNA curtain. Care must be taken to avoid injecting bubbles into the sample loop, and drop to drop connections should be made when changing any tubing connections.

\subsection{Prism-type two-color TIRFM}

DNA curtain experiments are performed with a custom-built prism-type two-color total internal reflection fluorescence microscope (TIRFM) based upon a Nikon inverted microscope, and details of the TIRFM have been described in detail elsewhere [25]. In brief, key features of the system include: (i) two iXon EMCCDs (Andor Technology); (ii) an autofocus setup (Nikon offset controller); (iii) a shutter controller (UNIBLITZ shutter driver, model VCM-D1); (iv) a flowcell heating system including an objective heater system (Bioptechs) and a flowcell heating system with a miniature benchtop controller (Omega). Data acquisition is performed with NISelements (Nikon Imaging Software, 64 bit 3.22.11(Build 728), LO). Two diode-pumped solidstate lasers (200 mW Coherent Sapphire) are used to excite different fluorophores, for example, a 488-nm laser is used for for RPA-eGFP, and a 561-nm laser is used for Atto565 [17]. Emission signals pass through a microscope objective $(60 \times, 1.2 \mathrm{NA}$, water immersion Plan Apo, Nikon), and a custom filter set (Chroma) [17], which separates the emissions of the two fluorophores allowing them to be imaged on two different iXon EMCCDs.

Tips \& Troubleshooting: The microscope objective is a water immersion type objective. For a data acquisition with a long time scale, the water drops between the flowcell and the objective evaporate easily, causing imaging and autofocus problems. To fix this issue use an immersion fluid (Immersol W (2010), Carl Zeiss) that has the same refractive index of water (1.334) but a lower evaporation rate.

\subsection{Photobleaching rates}

DNA curtains offer the potential for quantitative measures of binding lifetimes of ligands that interact with the Rad51 presynaptic complex, and we have used this approach extensively to measure interactions between presynaptic complexes and duplex DNA fragments labeled with a single Atto565 dye (Fig. 5) [42]. Before this data can be quantitated, one must first obtain an 
accurate measure of the photobleaching rates for the single Atto565 dyes using the following procedure:

Step 1. First, presynaptic complexes are formed on ssDNA curtains (see Section 4.7).

Step 2. Next, a 70-bp fully homologous dsDNA (10 nM) labeled with a single Atto565 dye is injected into the flowcell. Flow is terminated once the sample enters the flowcell (see Fig. 8), and the reactions are incubated for an additional 10 minutes to allow binding. This fully homologous substrate is used for photobleaching measurements because it binds very tightly to the presynaptic complex and the loss of signal corresponds primarily to photobleaching and not to substrate dissociation [42].

Step 3. Once the substrate is bound, the laser is turned on and a continuous data set (without shuttering) is acquired for a total of three minutes. Survival probability analysis of the resulting data set (see Section 5) yields the rate of photobleaching for that specific fluorophores under the laser illumination conditions used for the measurement (see examples in Fig. 5D and Fig. 6). Note that if the laser power is altered, then a new set of photobleaching data must be acquired to reflect the behavior of the fluorphore under the new illumination conditions.

The data obtained from the photobleaching experiment can also be used to verify that the observed binding events correspond to an interaction with a single Atto565 labeled dsDNA molecule. Specifically, events involving just one fluorophores tagged ligand should yield singlestep photobleaching trajectories, whereas events involving multiple dsDNA molecules will be revealed as two or more photobleaching steps (Fig. 6).

\section{Data acquisition and analysis of ssDNA curtains}

\subsection{Data acquisition and kymograph analysis}

NIS-elements (Nikon) is used to control data acquisition, and the resulting data (video format) is imported into Fiji (Software, ImageJ 1.48b, Wayne Rasband, National Institutes of Health, USA). The video data is then corrected for stage drift using the "StackReg" function in Fiji "Plugins". A kymogram is then generated for each individual presynaptic complex observed in the videos using the "Reslice" function in Fiji. The "Reslice" function saves the image information corresponding to a straight line superimposed on each ssDNA molecule, for every image frame of the video data, and then projects this image information as a function of time. The y-component of each kymograph reflects the position information of the molecules under observation, and $\mathrm{x}$-component represents time.

Analysis of the resulting kymograms can be used to determine information such as the timing and extent of presynaptic complex assembly and the binding of fluorescently tagged ligands to the presynaptic complex. For example, in the kymographs shown in Fig. 3B, the loss of RPAeGFP fluorescence signal over time reflects the displacement of RPA during the assembly of the wild-type Rad51 (dark) presynaptic complex. Analysis of this signal change can yield information on the kinetics of presynaptic complex assembly. Similarly, analysis of the kymograms can be used to reveal information related to ligand binding, such as dwell time and position distributions. For example, in Fig. 5C, each magenta line represents a fluorescently 
tagged dsDNA fragment bound to the unlabeled Rad51 presynaptic complex. The $\mathrm{x}$ - and $\mathrm{y}$-axis information reflecting the properties of each magneta line (i.e. dsDNA molecule) can be read and recorded by Fiji. The x-component information reflects the dwell time of the bound ligand (dsDNA), which can be used to determine the dissociation rate (Fig. 5E) by survival probability analysis (Section 5.2). The y-component information reflects the position of the bound dsDNA molecule along the Rad51-ssDNA presynaptic complex, which can be used to perform position distribution analysis [17]. The error bars for both the dwell time and the position distribution analysis can be estimated by bootstrap analysis (Section 5.3).

\subsection{Survival probability analysis}

Ligand binding parameters can be obtained using the following method to calculate and plot survival probability:

Step 1. First, the kymogram is divided into small time increments corresponding to [0 - $100 \mathrm{~ms}$, [100 - $200 \mathrm{~ms}$ ], [200 - $300 \mathrm{~ms}$ ], ..., [maximum dwell time - $100 \mathrm{~ms}$, maximum dwell time].

Step 2. We then calculate the percentage of all dwell times that are equal or greater than $100 \mathrm{~ms}$ for the first time window [0 - $100 \mathrm{~ms}$ ]. If all of the observed ligands remain bound during this first time interval then the result is $100 \%$ survival, and we then record: $\mathrm{x} 1=100 \mathrm{~ms}$, and $\mathrm{y} 1=100 \%$.

Step 3. Step 2 is then repeated for the next time interval [100 - $200 \mathrm{~ms}]$, and the data are recorded $\mathrm{x} 2=200 \mathrm{~ms}$ and a new y2 corresponding the fraction of remaining ligands.

Step 4. Step 2 is repeated until the last time interval is completed [maximum dwell time -100 ms, maximum dwell time].

Step 5. A plot of the entire $\mathrm{y}$ versus $\mathrm{x}$ data is then generated to obtain the survival probability versus time (Fig. 5D-E).

Step 6. The dissociation rate can then be calculated from the survival probability plots. For example, for a process with a single dissociation rate, as shown in Fig. 5E, the survival probability plot can be fitted with a single exponential function $-\mathrm{e}^{-k t}$, where $k$ represents the total dissociation rate, which is the sum of the real dissociation rate $k_{\text {real }}$ plus the rate of photobleaching $k_{\mathrm{p}}$ (see Section 4.8).

\subsection{Experimental error}

MATLAB (R2010a; MathWorks, Inc., Natick, MA) function 'bootci' (bootstrap confidence interval) is used to perform the bootstrap analysis to estimate the error for dwell time analysis and position distribution analysis. Two parameters are used: (i) Repeat, which is the number of bootstrap samples used in the computation (nboot); (ii) bootstrap confidence interval (100×(1alpha)). For example, 1000 repeat and $70 \%$ bootstrap confidence intervals are typically used for the error bars of dwell time analysis (Fig. 5D and 5E), and 1000 repeat and 95\% bootstrap confidence interval are used for the error bars of position distribution analysis [17].

\subsection{Microhomology analysis for homology search study}


Microhomology is an important concept for understanding our interpretation of the homology search mechanism (Section 3.4) [42]. Our definition of microhomology is a tract of nucleotide sequence of any given length within a dsDNA molecule that is complementary to a sequence within a ssDNA molecule. Within the context of our experiments, these molecules are the Atto565 labeled 70-bp dsDNA that is provided in solution and the M13-derived ssDNA that is anchored to the flowcell surface. To define these tracts of microhomology, we developed a MATLAB algorithm (MathWorks, Inc., Natick, MA) that scans all overlapping regions between the M13 ssDNA (M13mp18, 7,249 nt; N4040S NEB) and both strands of the 70-bp dsDNA substrate. In simple terms, the algorithm takes short windows (3-nt to 15-nt) of sequence information from both strands of the dsDNA and scans these sequences across the ssDNA define the location of all complementary regions (Fig. 7A). This section provides a brief overview of the algorithm, and here we use an 8-nt tract of microhomology on the top strand of the 70-bp dsDNA as an illustrative example (Fig. 7A).

Step 1. First, an index parameter $i$, is used to subdivide the top strand of the 70-bp dsDNA into all possible 8-nt segments. $\mathrm{T}(i: i+7)$ represents the 8 -nt sequences that will be scanned against the M13 ssDNA sequence (Fig. 7A). For instance, $i=1$ corresponds to the nucleotide segment encompassing positions 1 to 8 on the top strand of the dsDNA, $i=2$ corresponds to the nucleotide segment encompassing positions 2 to 9 on the top strand of the dsDNA, and so forth. A second index parameter $m$, is used to sequentially subdivide the M13 ssDNA into all possible 8-nt segments. M13( $m: m+7)$ represents all 8-nt windows on the M13 ssDNA sequence. For instance, $m=1$ corresponds to the segment on M13 defined by nucleotides 1-8, $m=2$ corresponds to the segment on M13 defined by nucleotides 2-9, $m=3$ corresponds to the segment on M13 defined by nucleotides 3-10, and so forth.

Step 2. For each value of $m$ that results in the identification of sequence microhomology, any nearby 8-nt tracts of microhomology are also identified by scanning a 70-nucleotide window on the M13 ssDNA for any additional 8-nt regions of homology that are complementary to the remainder of the 70-bp dsDNA.

Step 3. Steps 1 and 2 are repeated for each value of $i$ until reaching $i=63$, which corresponds to the full length of the 70-bp DNA, and the process is also repeated for the bottom strand of the dsDNA molecule.

Step 4. This entire procedure is then repeated for all desired lengths of microhomology (see Fig. 7B for a typical example).

\section{Conclusion}

Single-stranded DNA curtains provide a unique experimental platform for direct visualization of Rad51 presynaptic complexes, offering the potential for new insights into the assembly, stability and regulation of this key recombination intermediate. The procedures described here can be adapted to study many different questions related to homologous DNA recombination, and one can envision future studies directed towards the use of long duplex DNA fragments to gain further insights into the homology search and strand invasion reactions, reactions incorporating the many different recombination accessory proteins, and experiments designed to determine how nucleosomes and chromatin affect the homology search and strand invasion. The methods 
described here may also be particularly amendable to studies of the many helicase and motor proteins associated with recombination, including Srs2, Sgs1, Rad54, and Mph1

\section{Acknowledgments}

We thank members of the Greene laboratory, especially Kyle Kaniecki, Corentin Moevus, Chu Jian Ma, Justin Steinfeld, Dr. Fabian Erdel, Dr. Johannes Stigler, and Daniel Duzdevich for comments on the manuscript. This research was funded by NIH grants GM082848 and GM074739 (E.C.G), and by the National Science Foundation grant MCB1154511 (E.C.G.).

\section{Figure Legends}

Fig. 1. Simplified overview of homologous recombination. Upon formation of a DSB the broken ends of the chromosome (green) are processed to yield long 3' ssDNA overhangs. These overhangs are bound by RPA (orange), which protects the ssDNA and removes secondary structure. RPA is then replaced by the recombinase protein Rad51 (blue), which forms a long right-handed filament on the ssDNA referred to as the presynaptic complex; for clarity only one of the DNA ends is shown after assembly of the presynaptic complex assembly step. The Rad51 presynaptic complex must the search for a homologous dsDNA molecule (shown in black) and pair the presynaptic ssDNA (green) with the complementary strand in the homolog.

Fig. 2. Overview of single-stranded DNA curtains. (A) Overview of the rolling circle replication assay is used to make long ssDNA substrates that are biotinylated at the 5' ends. (B) Schematic illustration of a double-tethered ssDNA curtain and presynaptic complex assembly. The slide surface is coated with a lipid bilayer and the ssDNA is anchored to the bilayer through a biotinstreptavidin linkage. The ssDNA (blue line) is pushed into chromium barriers, extended in the presence of RPA-eGFP (green) and the ends of the RPA-ssDNA complexes are anchored to exposed pedestals through nonspecific adsorption. RPA is then replaced with Rad51 (blue) allowing for the assembly of the presynaptic complexes. Adapted with permission from Ref. 17.

Fig. 3. Visualizing single-stranded DNA curtains. (A) Wide-field TIRFM image of ssDNA curtains bound by RPA-eGFP. (B-F) Kymographs depicting the assembly of wild-type (unlabeled) Rad51 presynaptic complexes on individual molecules of ssDNA bound the RPAeGFP. Loss of the eGFP signal upon injection of Rad51 and ATP reflects the displacement of RPA-eGFP from the ssDNA by unlabeled Rad51. Free Rad51 is removed during the first chase by injecting buffer that contains free RPA-eGFP and the indicated nucleotide cofactor (ATP, no nucleotide, ATP $\gamma$, ADP, or AMP-PNP, as indicated). In all panels, the nucleotide cofactors are removed during the second chase. The reappearance of the RPA-eGFP signal reflects disassembly of the Rad51 presynaptic complexes. (G) Quantitation of the signal intensity versus time for each of the indicated chase conditions. Adapted with permission from Ref. 17.

Fig. 4. Rad52 interactions with the RPA-ssDNA complexes. (A) Schematic illustration of experiment designed to look for interactions between ssDNA bound by RPA-mCherry (magenta) and SNAP-tagged Rad52 labeled with Alexa 488 (green). (B) Wide-field TIRFM image of a 
ssDNA curtain bound by RPA-mCherry in the absence (top panel) and presence of $\mathrm{SNAP}_{488^{-}}$ Rad52 (bottom panel). (C) Images highlighting a single ssDNA molecule showing the SNAP $488^{-}$ Rad52 channel only, the RPA-mCherry channel only, and an overlay of the two images. (D) Kymographs illustrating the real time association of $\mathrm{SNAP}_{488}-\mathrm{Rad}_{52}$ with either RPA-mCherry (top panel) or wild type (unlabeled) RPA (bottom panel) bound to ssDNA. Adapted with permission from Ref. 32.

Fig. 5. Duplex DNA sampling and capture by the Rad51 presynaptic complex. (A) Schematic showing the general strategy for detecting interactions between fluorescently tagged dsDNA fragments and the Rad51 presynaptic complexes. Transient interactions are detected by injecting the dsDNA and visualizing the reactions at high frame rates while free dsDNA is within the flowcell. Stable interactions can be detected by flushing away all of the free dsDNA fragments and visualizing only those dsDNA molecules that remain bound to the presynaptic complex. (B) Kymograph showing examples of transient interactions. (C) Kymograph showing examples of stable interactions. Note the different time scales bars in (B) and (C). (D) Survival probability plot for transient binding intermediates. A plot of photobleaching is shown for comparison. (D) Survival probability plot for a stable binding intermediated. Adapted with permission from Ref. 17.

Fig. 6. Photobleaching analysis. (A) Photobleaching trajectories for 70-bp dsDNA fragments labeled with Atto565 and stably bound to the Rad51 presynaptic complex. Examples of 1-, 2and 3-step photobleaching are depicted. (B) Relative occurrence of 1-, 2- and 3-step photobleaching steps. (C) Survival probability plot for the 1-step photobleaching trajectories.

Fig. 7. Microhomology analysis. (A) Overview of the algorithm used to analyze sequence microhomology. The two dsDNA strands (top and bottom) are subdivided into short segments of the desired length typically ranging from 3- to 10-nucletides (in this example 8 nucleotides). Each of these segments is scanned in across the M13 ssDNA sequence to identify regions of perfect sequence complementary (i.e. microhomology). (B) Examples of microhomology analysis graphs for the top and bottom strands of a typical 70-bp dsDNA substrate. Each bar represents a different length of microhomology ranging from 3 to 10 nucleotides (x-axis). The $\mathrm{N}$ value corresponds to the total number of microhomology tracts of a given length that were found M13. For instance, in the top strand $\mathrm{N}=7105$ for a microhology length of 3 nucleotides means that that there were a total of 7105 sites within the M13 substrate that were complementary to all possible 3-nt segments within the top strand of the dsDNA. The y-axis represents the average number of such sites within a 70-bp window. So there were an average of $\sim 3$ positions within all possible 70-bp windows, which contained 3-nt of microhomology. In contrast, $\mathrm{N}=1$ for 9 -nt of microhomology, meaning that there was only a single position within the entire M13 ssDNA that was complementary to 9 contiguous nucleotides within the top strand of the dsDNA molecule. (C) Shows a color-coded schematic showing the locations of all 8-nt sequences within the dsDNA fragment (colored bars) that complementary to 8-nt sequences of microhomology on the M13 ssDNA (colored arrows). Adapted with permission from Ref. 17. 
Fig. 1

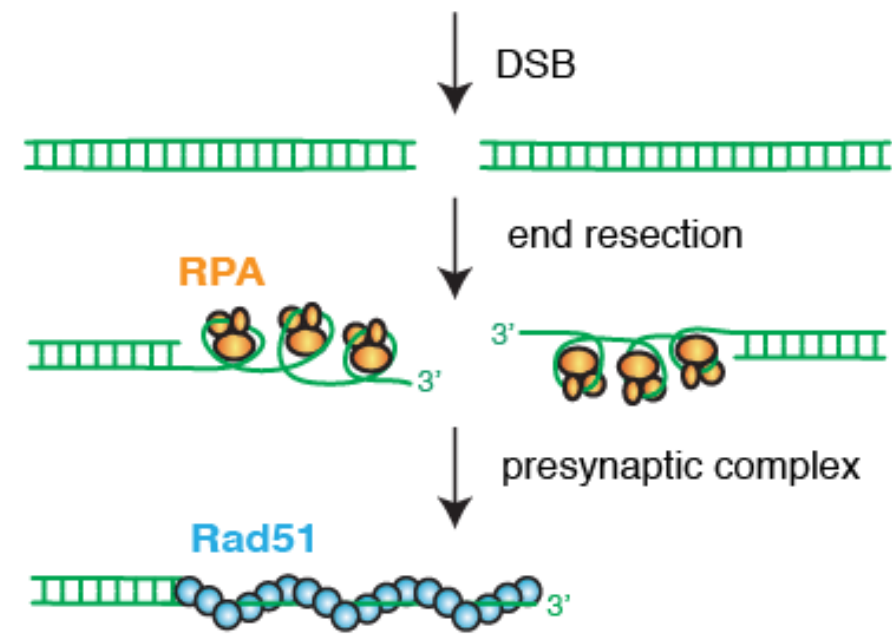



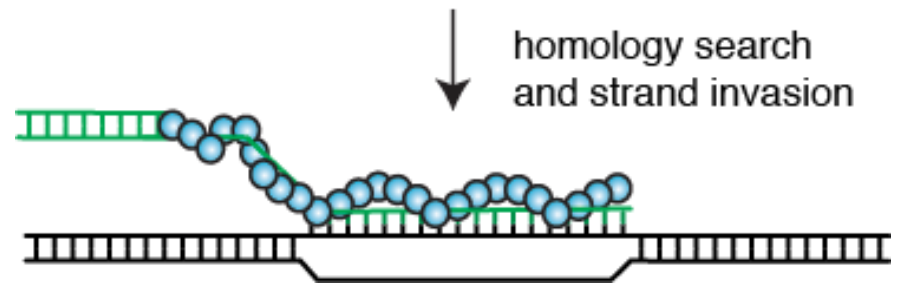


Fig. 2

A

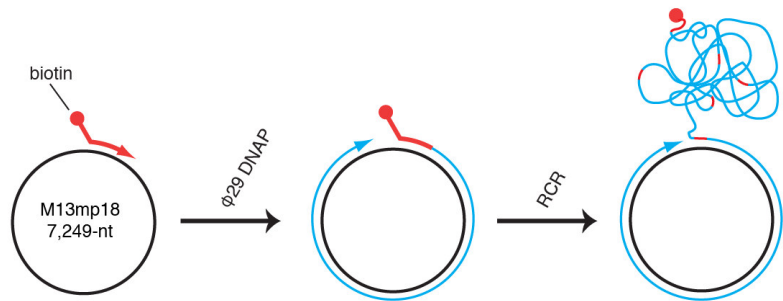

B

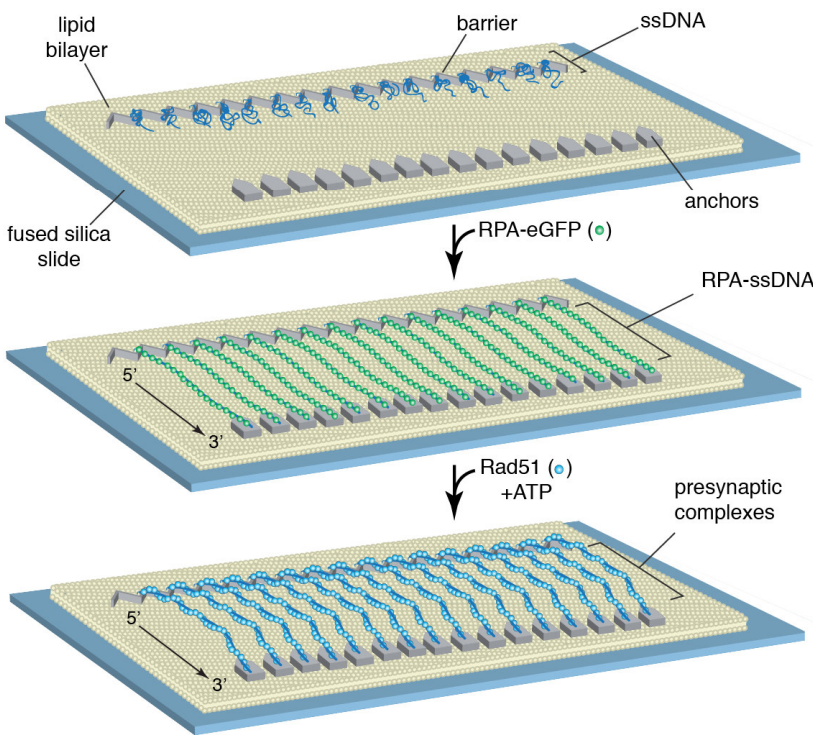


Fig. 3

A
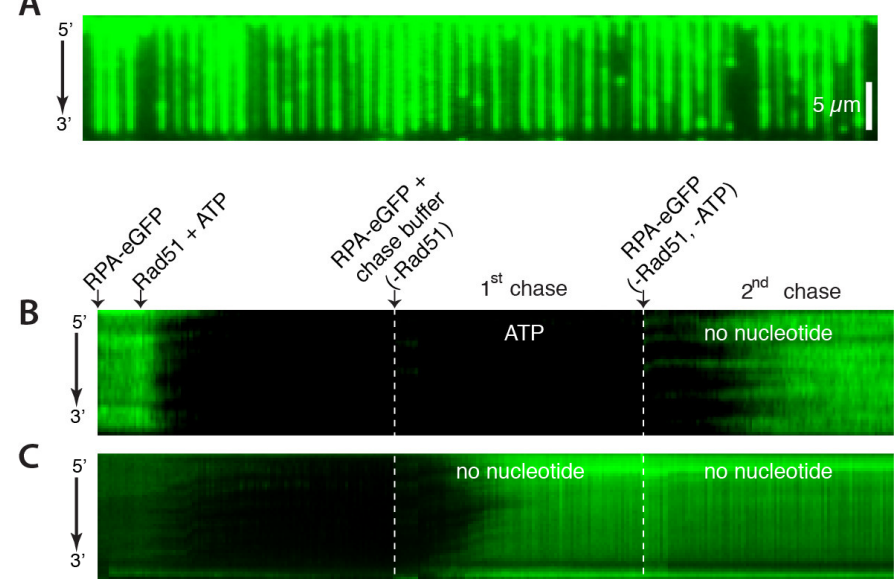

D

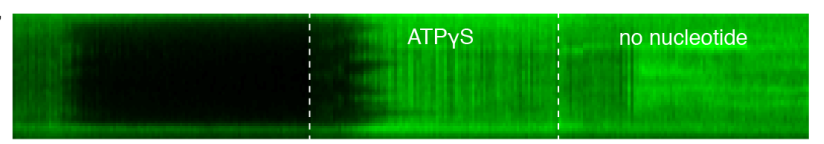

E

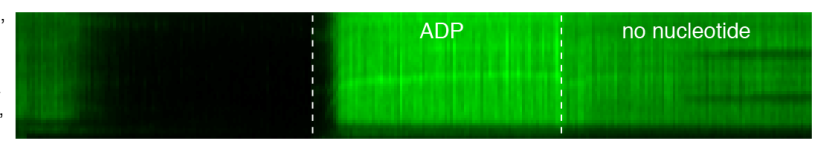

F
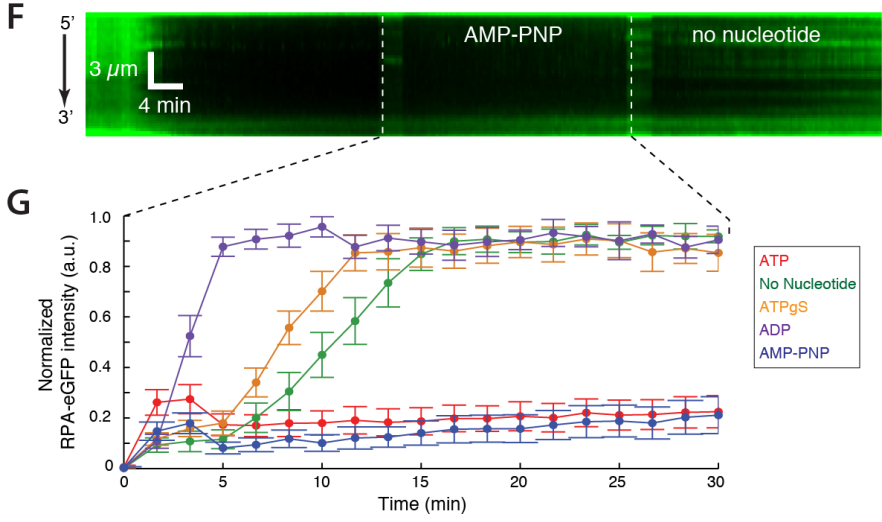
Fig. 4

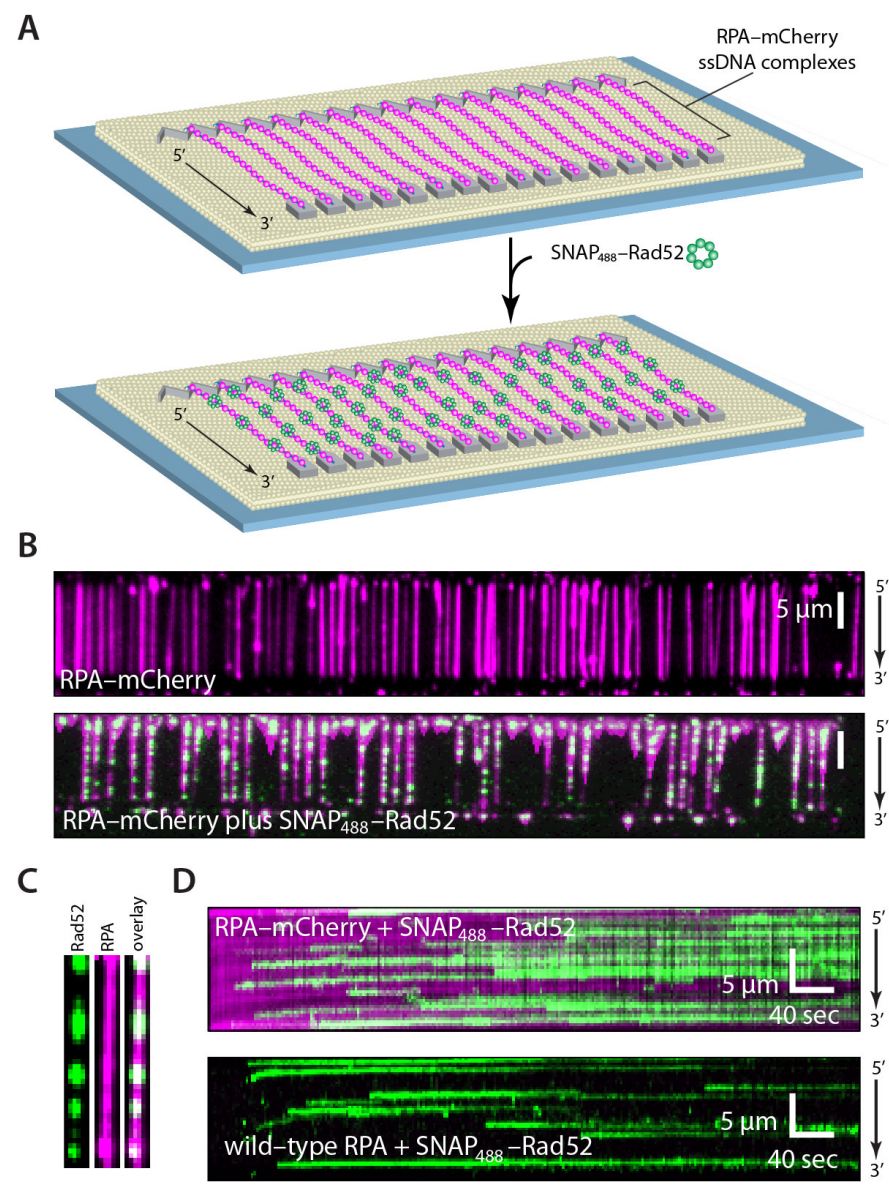


Fig. 5

A

transient sampling

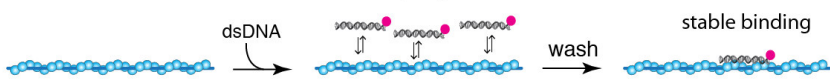

B

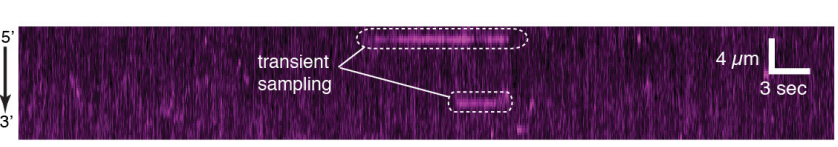

C

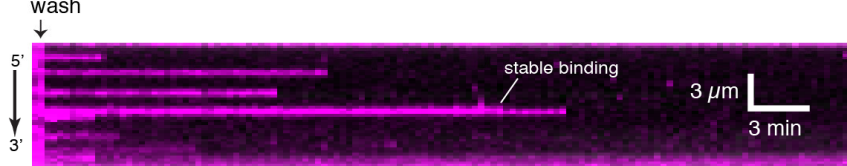

D

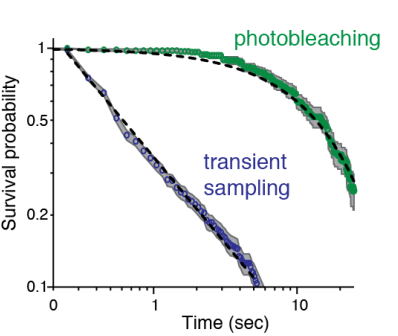

E

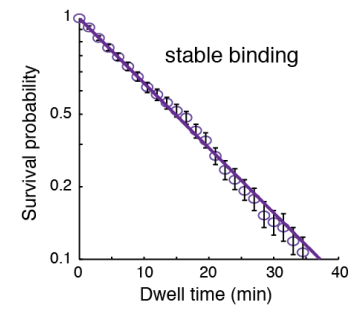


Fig. 6
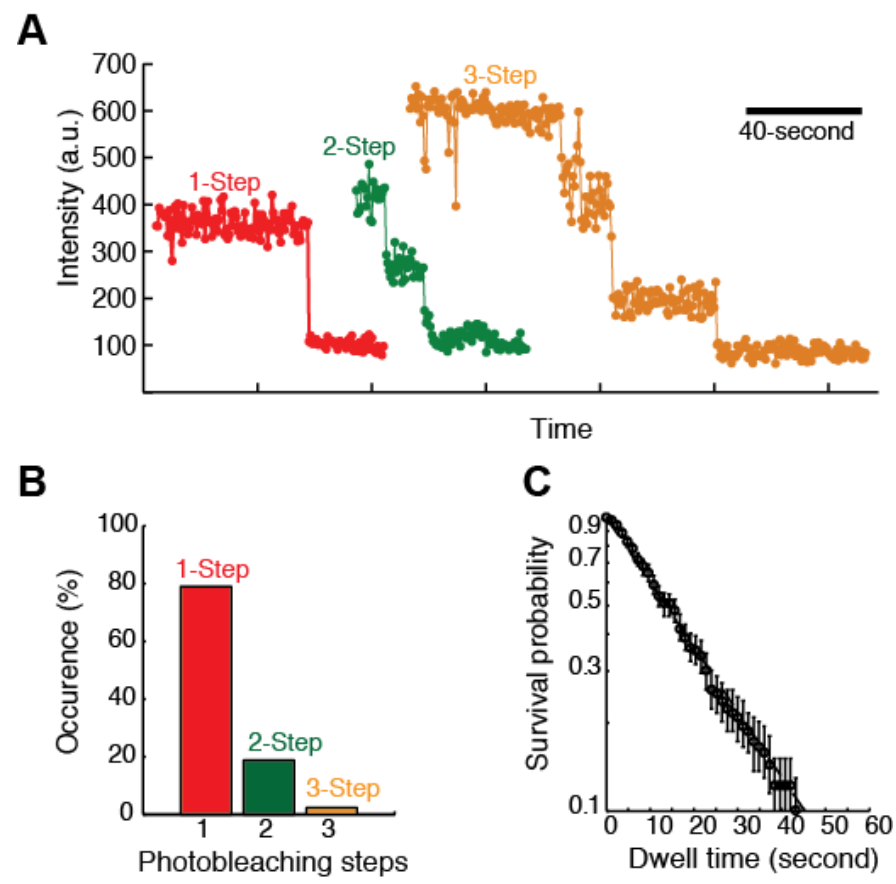
Fig. 7
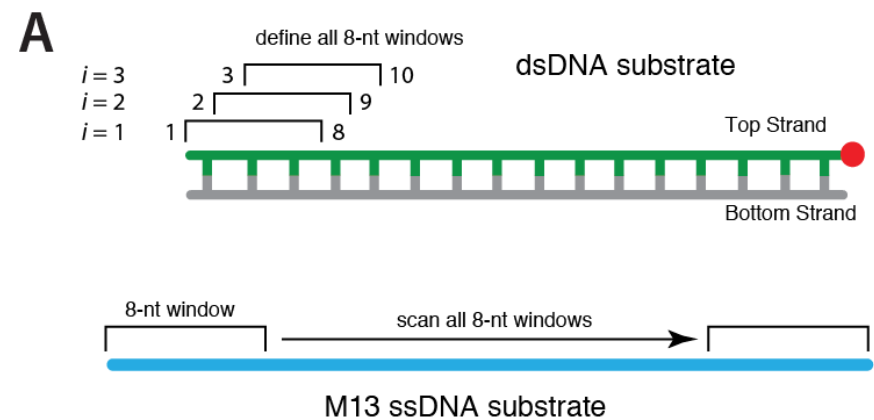

B
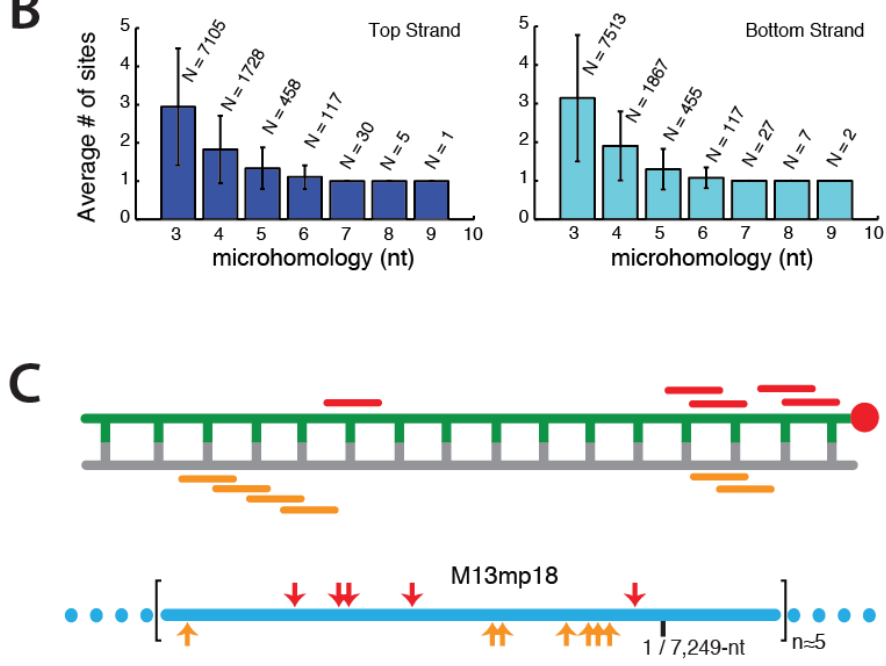


\section{References}

1.De Bont, R. and N. van Larebeke, Endogenous DNA damage in humans: a review of quantitative data, Mutagenesis, 2004. 19(3): p. 169-185.

2.Harper, J.W. and S.J. Elledge, The DNA damage response: Ten years after, Molecular Cell, 2007. 28(5): p. 739-745.

3.Helleday, T., E. Petermann, C. Lundin, B. Hodgson, and R.A. Sharma, DNA repair pathways as targets for cancer therapy, Nature Reviews Cancer, 2008. 8(3): p. 193-204.

4.Sung, P. and H. Klein, Mechanism of homologous recombination: mediators and helicases take on regulatory functions, Nature Reviews Molecular Cell Biology, 2006. 7(10): p. 739-750.

5.Bianco, P.R., R.B. Tracy, and S.C. Kowalczykowski, DNA strand exchange proteins: a biochemical and physical comparison, Front Biosci, 1998. 3: p. D570-603.

6.Cromie, G.A., J.C. Connelly, and D.R. Leach, Recombination at double-strand breaks and DNA ends: conserved mechanisms from phage to humans, Mol Cell, 2001. 8(6): p. 1163-74.

7.Morrical, S.W., DNA-Pairing and Annealing Processes in Homologous Recombination and Homology-Directed Repair, Cold Spring Harb Perspect Biol, 2015. 7(2): p. a016444.

8.Renkawitz, J., C.A. Lademann, and S. Jentsch, DNA damage Mechanisms and principles of homology search during recombination, Nature Reviews Molecular Cell Biology, 2014. 15(6): p. 369-383.

9.Barzel, A. and M. Kupiec, Finding a match: how do homologous sequences get together for recombination?, Nat Rev Genet, 2008. 9(1): p. 27-37.

10.San Filippo, J., P. Sung, and H. Klein, Mechanism of eukaryotic homologous recombination, Annu Rev Biochem, 2008. 77: p. 229-57.

11.Heyer, W.D., K.T. Ehmsen, and J. Liu, Regulation of homologous recombination in eukaryotes, Annu Rev Genet, 2010. 44: p. 113-39.

12.Symington, L.S., R. Rothstein, and M. Lisby, Mechanisms and regulation of mitotic recombination in Saccharomyces cerevisiae, Genetics, 2014. 198(3): p. 795-835.

13.Cox, M.M., Historical overview: searching for replication help in all of the rec places, Proc Natl Acad Sci U S A, 2001. 98(15): p. 8173-80.

14.Cox, M.M., Regulation of bacterial RecA protein function, Crit Rev Biochem Mol Biol, 2007. 42(1): p. 41-63.

15.Chen, Z., H. Yang, and N.P. Pavletich, Mechanism of homologous recombination from the RecA-ssDNA/dsDNA structures, Nature, 2008. 453(7194): p. 489-4. 
16.Lee, J.Y., T. Terakawa, Z. Qi, J.B. Steinfeld, S. Redding, Y. Kwon, W.A. Gaines, W. Zhao, P. Sung, and E.C. Greene, DNA RECOMBINATION. Base triplet stepping by the Rad51/RecA family of recombinases, Science, 2015. 349(6251): p. 977-81.

17.Qi, Z., S. Redding, J.Y. Lee, B. Gibb, Y. Kwon, H. Niu, W.A. Gaines, P. Sung, and E.C. Greene, DNA sequence alignment by microhomology sampling during homologous recombination, Cell, 2015. 160(5): p. 856-69.

18.Bustamante, C., Z. Bryant, and S.B. Smith, Ten years of tension: single-molecule DNA mechanics, Nature, 2003. 421(6921): p. 423-7.

19.King, G.A., P. Gross, U. Bockelmann, M. Modesti, G.J. Wuite, and E.J. Peterman, Revealing the competition between peeled ssDNA, melting bubbles, and S-DNA during DNA overstretching using fluorescence microscopy, Proc Natl Acad Sci U S A, 2013. 110(10): p. 3859-64.

20.van Mameren, J., P. Gross, G. Farge, P. Hooijman, M. Modesti, M. Falkenberg, G.J. Wuite, and E.J. Peterman, Unraveling the structure of DNA during overstretching by using multicolor, single-molecule fluorescence imaging, Proc Natl Acad Sci U S A, 2009. 106(43): p. 18231-6.

21.Candelli, A., M. Modesti, E.J. Peterman, and G.J. Wuite, Single-molecule views on homologous recombination, $Q$ Rev Biophys, 2013. 46(4): p. 323-48.

22.Forget, A.L. and S.C. Kowalczykowski, Single-molecule imaging brings Rad51 nucleoprotein filaments into focus, Trends Cell Biol, 2010. 20(5): p. 269-76.

23.Hilario, J. and S.C. Kowalczykowski, Visualizing protein-DNA interactions at the singlemolecule level, Curr Opin Chem Biol, 2010. 14(1): p. 15-22.

24.Spies, M., There and back again: new single-molecule insights in the motion of DNA repair proteins, Curr Opin Struct Biol, 2013. 23(1): p. 154-60.

25.Greene, E.C., S. Wind, T. Fazio, J. Gorman, and M.L. Visnapuu, DNA curtains for highthroughput single-molecule optical imaging, Methods Enzymol, 2010. 472: p. 293-315.

26.Fazio, T., M.L. Visnapuu, S. Wind, and E.C. Greene, DNA curtains and nanoscale curtain rods: high-throughput tools for single molecule imaging, Langmuir, 2008. 24(18): p. 10524-31.

27.Graneli, A., C.C. Yeykal, T.K. Prasad, and E.C. Greene, Organized arrays of individual DNA molecules tethered to supported lipid bilayers, Langmuir, 2006. 22(1): p. 292-9.

28.Gibb, B., T.D. Silverstein, I.J. Finkelstein, and E.C. Greene, Single-stranded DNA curtains for real-time single-molecule visualization of protein-nucleic acid interactions, Anal Chem, 2012. 84(18): p. 7607-12.

29.Nguyen, B., J. Sokoloski, R. Galletto, E.L. Elson, M.S. Wold, and T.M. Lohman, Diffusion of human replication protein A along single-stranded DNA, J Mol Biol, 2014. 426(19): p. 3246-61. 
30.Wold, M.S., Replication protein A: a heterotrimeric, single-stranded DNA-binding protein required for eukaryotic DNA metabolism, Аnnu Rev Biochem, 1997. 66: p. 61-92.

31.Deng, S.K., B. Gibb, M.J. de Almeida, E.C. Greene, and L.S. Symington, RPA antagonizes microhomology-mediated repair of DNA double-strand breaks, Nat Struct Mol Biol, 2014. 21(4): p. $405-12$.

32.Gibb, B., L.F. Ye, Y. Kwon, H. Niu, P. Sung, and E.C. Greene, Protein dynamics during presynaptic-complex assembly on individual single-stranded DNA molecules, Nat Struct Mol Biol, 2014. 21(10): p. 893-900.

33.Gorman, J., T. Fazio, F. Wang, S. Wind, and E.C. Greene, Nanofabricated racks of aligned and anchored DNA substrates for single-molecule imaging, Langmuir, 2010. 26(2): p. 1372-9.

34.Sung, P. and H. Klein, Mechanism of homologous recombination: mediators and helicases take on regulatory functions, Nat Rev Mol Cell Biol, 2006. 7(10): p. 739-50.

35.Bernstein, K.A., S. Gangloff, and R. Rothstein, The RecQ DNA helicases in DNA repair, Annu Rev Genet, 2010. 44: p. 393-417.

36.Symington, L.S. and W.D. Heyer, Some disassembly required: role of DNA translocases in the disruption of recombination intermediates and dead-end complexes, Genes Dev, 2006. 20(18): p. 2479-86.

37.Taylor, M.R., M. Spirek, K.R. Chaurasiya, J.D. Ward, R. Carzaniga, X. Yu, E.H. Egelman, L.M. Collinson, D. Rueda, L. Krejci, and S.J. Boulton, Rad51 Paralogs Remodel Pre-synaptic Rad51 Filaments to Stimulate Homologous Recombination, Cell, 2015. 162(2): p. 271-86.

38.Gaines, W.A., S.K. Godin, F.F. Kabbinavar, T. Rao, A.P. VanDemark, P. Sung, and K.A. Bernstein, Promotion of presynaptic filament assembly by the ensemble of S. cerevisiae Rad51 paralogues with Rad52, Nat Commun, 2015. 6: p. 7834.

39.Liu, J., L. Renault, X. Veaute, F. Fabre, H. Stahlberg, and W.D. Heyer, Rad51 paralogues Rad55-Rad57 balance the antirecombinase Srs2 in Rad51 filament formation, Nature, 2011. 479(7372): p. 245-8.

40.Gibb, B., L.F. Ye, S.C. Gergoudis, Y. Kwon, H. Niu, P. Sung, and E.C. Greene, Concentration-dependent exchange of replication protein A on single-stranded DNA revealed by single-molecule imaging, PLoS One, 2014. 9(2): p. e87922.

41.Sugiyama, T., E.M. Zaitseva, and S.C. Kowalczykowski, A single-stranded DNA-binding protein is needed for efficient presynaptic complex formation by the Saccharomyces cerevisiae Rad51 protein, Journal of Biological Chemistry, 1997. 272(12): p. 7940-7945.

42.Qi, Z., S. Redding, J.Y. Lee, B. Gibb, Y. Kwon, H.Y. Niu, W.A. Gaines, P. Sung, and E.C. Greene, DNA Sequence Alignment by Microhomology Sampling during Homologous Recombination, Cell, 2015. 160(5): p. 856-869. 